\author{
Research Article
}

\title{
Determination of the whitten effect based on vaginal cell characteristics, vulva appearance and behavior in grouped female mice
}

\author{
Nur Hannan Zakaria and Sabrina Sukardi* \\ Department of Biomedical Science, Faculty of Medicine and Health Sciences, Universiti Putra Malaysia, 43400 Serdang, Selangor Darul Ehsan, Malaysia. \\ https://doi.org/10.28916/lsmb.3.8.2019.56 \\ Received 10 December 2019, Revisions received 20 December 2019, Accepted 22 December 2019, Available online 30 December 2019
}

\begin{abstract}
Introduction: Pheromones are chemicals produced by an animal that affects the behavior of another animal or the same species. Information conveyed includes location, presence of food or threat, sexual attraction, courtship, and dam-pup interactions. Pheromones are used widely in laboratory mice facilities to synchronize estrus and simultaneous breeding for logistic purposes. Female mice housed together in the absence of the male exhibit the Lee-Boot effect of lengthened diestrus or ovarian inactive period of up to several weeks. Whitten effect is described when a large number of female mice housed together in the absence of the male and having diestrus, will enter estrous 48 to 72 hours later upon exposure to male odors or male mouse urine soaked-bedding. Objective: The aim of this study is to determine the time taken for the Whitten effect to occur based on changes in vaginal cell characteristics, vulva appearance and behavior in grouped female mice. Methodology: Ten female mice were acclimatized to the animal facility for 3 estrus cycles or 12 days. Phases of the estrus cycle were evaluated by visual observation to assess changes to the vulva and vaginal cytology. Male urine soaked-bedding were exposed to females for 3 days and the time taken for the Whitten effect to occur was determined based on changes in vaginal cell characteristics, vulva appearance and observation of behavior. Result: The Cochran's Q test was used to observe the changes from diestrus to proestrus and later estrus. The results showed a significant difference $(\mathrm{p}<0.05)$ in the number of mice that successfully enter the proestrus and estrus phases over a four time point, $\chi^{2}(2)=$ 18.857. Conclusion: The Whitten effect occurs after 72 hours of exposure to male urine soaked-bedding based on vulva appearance, vaginal cell characteristics and behavior in grouped female mice.
\end{abstract}

Keywords: Whitten effect, female mice, pheromone, vaginal cell characteristics, behaviour

\subsection{Introduction}

Laboratory mice are useful models for research in reproduction because of its inherent fecundity and small size (Bertoli and Murphy, 2014). Female mice are polyestrous mammals and ovulate spontaneously. The short-term duration of the estrus cycle of only 4 to 5 days renders these species favourable as an ideal animal model for studies of changes during the reproductive cycle. Throughout the estrus cycle, the hypothalamic-pituitary-gonadal (HPG) axis was involved in a variety of changes for puberty, development, and senescence of the estrus cycle. The vaginal mucosa experiences massive structural modifications and is influenced by hormones such as follicle-stimulating hormone (FSH) which enhances the development and growth of ovarian follicles, luteinizing hormone (LH) which controls the hormones of estrogen and progesterone, as well as progesterone and estradiol during this estrous cycle (Aritonang et al., 2017). The neural network directing the hypothalamus as well as the cell synthesizing and emitting gonadotropin-releasing hormone $(\mathrm{GnRH})$ with their regulatory neurotransmitters, controls this natural progression (Maffucci and Gore, 2009).

The female mouse estrus cycle is divided into four phases: proestrus, estrus, metestrus, and diestrus (Gonzalez, 2016). These various phases are identified by investigating vagina washes or cell smears that are vital for determining receptive females for artificial insemination, establishing mating and analysing the female reproductive tract's phenotype. Sample from mice in proestrus, mainly presented nucleated cells with occasional leukocytes, usually takes 12 hours and the mouse will start showing physical signs of male acceptance. Estrus, distinguished by densely keratinized, cornified anucleated cells last for 12 hours with maximum male acceptance, females remaining silent when touching the back and exhibiting lordosis (Gonzalez, 2016). This is followed by metestrus, which comprised poorly stained cornified cells as well as leukocytes and nucleated cells, lasting 21 hours without male acceptance and eventually diestrus, where a large number of leucocytes with mucus strands are visible and last 57 hours (Macdonald et al., 2014). The vulva appearance technique which defined the different phases of the female mice estrus cycle is comparatively faster, simpler and more precise than the technique of vaginal cytology (Ekambaram, et. al., 2017). Swollen, moist, pink tissue describes the vaginal opening of mice in proestrus. The opening is broad and the dorsal and ventral edges often have wrinkles or striations.

\footnotetext{
* Correspondence: Department of Biomedical Science, Faculty of Medicine and Health Sciences, Universiti Putra Malaysia, 43400 Serdang, Selangor Darul Ehsan, Malaysia.

e-Mail: sabrina@upm.edu.my
}

(C) 2019 by the Author(s). Life Sciences, Medicine and Biomedicine (ISSN: 2600-7207) Published by Biome Journals. Wholly owned by Biome Scientia Sdn Bhd., previously by WWH Networks Sdn Bhd. Attribution-ShareAlike 4.0 International (CC BY-SA 4.0). This open access article is distributed based on the terms and conditions of the Creative Commons Attribution license https://creativecommons.org/licenses/by-sa/4.0/ 
In estrus, the vaginal opening becomes less pink, less moist, and less swollen. Metestrus has a vaginal opening that is not wide open, not swollen, and white cellular debris may be noticeable. The vaginal opening in diestrus is relatively small and closed without swelling of the tissue (Byers et al., 2012).

Mate preference and copulatory behavior are based on pheromones and synchronized with ovulation to ensure reproductive success in female rodents (Hellier et al., 2018). Exposure of a mixture of male mice pheromones to female mice triggers female olfactory preferences and speeds up the vaginal opening (Thoß et al., 2019). Karlson and Luscher in 1959 define pheromone as a volatile chemical that is tiny (5 to 17 of carbon atoms), either signalling or releasing (immediate response) or priming type (response takes a long time to occur), generated by a species that influences the same species' behavior or physiology. Pheromone sources may be from skin glands, faeces, saliva, respiratory breathing or breath, vaginal secretions, and urine. Pheromones are detected by the vomeronasal organ (VNO) located at the base of the nasal septum (Vanderburgh, 2008 ; Haga et. al., 2010). Examples of pheromones are copulin in vaginal secretions which attract males (Grammer et. al., 2004), androgen-like substances in male mice urine affects female mice estrus cycles (Haga et. al., 2010) and androstenol and androstenone in the saliva of boars attracts sows (Wani et. al., 2013). Pheromone elements in mice include both volatile organic compounds and non-volatile proteins, such as exocrine gland-secreted peptide 1 (ESP1), which is secreted by tear glands (Bind et. al., 2013) and darcin (MUP20), a member of the major urinary proteins (MUPs) (Roberts et. al., 2010).

There are a few MUPs found in male mice urine that is shown to promote female attraction, conditioned place preference, malemale territorial aggression or play a role in signalling individual identity (Chamero et. al., 2007; Roberts et. al., 2010; Roberts et. al., 2012 and Rodriguez, 2016). Pheromones are important in mice reproduction and four effects were observed: Lee-Boot Effect, Whitten Effect, Bruce Effect and Hoover-Drickamer Effect. These olfactory stimuli cause significant changes in the female mouse estrous cycle. Grouping female mice causes the rhythm of the estrus cycle to be interrupted. This usually occurs in a large animal breeding facility where space restrictions are imposed. The suppression of the female mouse estrous cycle and an anestrus or pseudo-pregnant state occurs when females are housed in groups in absence of the male mice and this phenomenon is known as the Lee-Boot effect (Dalal et al., 2001). This suppression of estrus is an advantage for logistic purposes as breeding can be synchronized with the Whitten effect. According to Carlson (2013), the pheromones in male mice urine may induce estrous synchronization (Whitten effect) as these female mice will enter estrus simultaneously when exposed to male mice urine-soaked bedding. The objective of this study is to determine the time taken for the Whitten effect to occur whether, in hours or days where vulval appearance, vagina cell characteristics and behavioural changes from an anestrus or prolong diestrus phase advance to proestrus and estrus phase. This study will serve as a recommendation for mice breeders on methods to synchronize estrus in their female mice colony for better breeding management.

\subsection{Materials and methods}

\section{$2.1 \quad$ Ethical approval}

The animal ethics study protocol was authorized by the Institutional Animal Care and Use Committee (IACUC) with the reference No. 00222, where all protocols adhered strictly as approval in Universiti Putra Malaysia (UPM).

\subsection{Animal experimentation}

Nine-week-old sexually matured female ICR mice obtained from the UPM animal house were used to conduct this experiment. This group of female mice were kept in a standard clean mice cage in a colony room with pine shaving bedding for 12 hours light and 12 hours darkness throughout the period of the study with food and water ad libitum.

\section{Determination of the phases of the estrus cycle}

Rrepresentative photographs were acquired for each phase of the estrus cycle following these steps, firstly, a visual observation by assessing the vulva appearance of each mouse and secondly, vaginal cytology to verify the phase of the estrus cycle (Byers et al., 2012). This method objective is to record the degree of variation within the phases of the estrus cycle among cycling mice inside a group (Dalal et al., 2001). Ten female mice were permitted to acclimatize for up to 3 estrus cycle (12 days) to the environment of the animal facility. Each female mouse was marked with black permanent ink on their tails.

\section{Visual observation of vulva}

In this method, the vulva of each female mouse was carefully observed under adequate illumination within the investigation room because light sources are necessary for visual examination (Yoshinaka et al., 2017). Each mouse was held using the non-dominant hand by the tail with the fore-paws resting on a cage lid. The tail was gently raised to assess the vulva by the method explained by Champlin, et al. (1973). The redness or swelling of the vulva was observed.

\section{Collection and analyses of vaginal smears}

Vaginal cytology samples were collected early in the morning after turning on the lights. Lavage generally produces a higher sample of cells. The accurate interpretation of vaginal cytology samples depends on the quality of the sample preparation, regardless of the methodology used. In this method, the animal was held gently in the palm of the hand with the index finger securing the tail. A drop of saline was placed at the entrance of the vaginal to a depth of 2 to $5 \mathrm{~mm}$ using a blunt end disposable pipette in order to prevent contact with the vagina. The vaginal wall was scraped gently and the saline re-aspirated. The step was repeated 4 to 5 times using the same pipette to obtain a sufficient number of cells in a single sample. The aspirated saline was expelled onto a clean glass slide and smeared at room temperature using the tip of the pipette to help evaporation (Mamrot et al., 2015). Slides were labelled with the female mice identification numbers, the date, and study number. The slides were completely air dried and then flooded with $1 \mathrm{ml}$ of Methylene Blue for 60 seconds to stain the nucleus of epithelial cells and leukocytes. The excess Methylene Blue was discarded, and the slide rinsed gently in water and tap to dry. The slides were viewed under a light microscope at $40 \mathrm{X}$ objective. The vaginal smears were collected daily from each female for 12 days, for accurate determination of the phases of the estrus cycle. 
This method was used to identify the effect of male mice urine on the female estrus cycle. Ten female mice were allowed to acclimatize for 12 days in order to encourage the transition into anestrus or prolong the phase of diestrus. The female mice were exposed to 75 to $100 \mathrm{ml}$ of 7-day-old urine-soaked bedding from a cage containing 6 to 10 male ICR mice aged 6 to 7 weeks old. This method leads to exposure to airborne olfactory signs without male mice. These female mice groups were exposed for 24,72 , or 96 hours to male-urine-soaked bedding (Dalal et al., 2001). The male urine soaked-bedding was placed in wraps and hung outside the female cage from a retort stand.

\subsection{Determining the Whitten effect}

\subsubsection{Identification of behavior}

The female mice behavior were observed after exposure to the urine-soaked bedding of male mice for 3 days at 9 am until $12 \mathrm{pm}$. Changes in behavior like mounting other females, lordosis or excitement, increase activity and making noises are some of the changes that can be observed.

\subsubsection{Evaluation of vulva appearance and vaginal cell characteristics}

Vaginal smears, the appearance of the vulva and behavioural estrus were examined from the time of introduction of male urinesoaked bedding until the third day. The time taken for the changes from anestrus or prolong diestrus to proestrus and estrus may occur between 1 to 3 days of exposure.

\section{$2.8 \quad$ Statistical analysis}

SPSS version 20 was used for the data analysis. Statistical analysis of data on the changes from diestrus to proestrus and later estrus were examined with Cochran's Q test. Results were expressed as Chi-Square $\left(\mathrm{X}^{2}\right)$ and $\mathrm{P}<0.05$ was accepted for statistical significance.

\subsection{Results}

A wide variation of the estrous cycle phases was identified among the ten female mice housed in a group and isolated from male mice for 12 days after acclimatization to the animal facilities. For each female mouse, the relative ratio of each cell type was utilized to evaluate each of the estrus phases. Proestrus, estrus, metestrus and diestrus are the normal arrangement of the estrous cycle phases.

\subsection{Lee Boot effect}

Table 1 showed that two female mice were in proestrus, five female mice in estrus, one female mouse in metestrus and two female mice in diestrus phase on day 1 of acclimatization to the animal facility as determined by the cytological assessment of vaginal smears. The number of mice entering the diestrus phase increases from two female mice to five female mice after two days. After 12 days of acclimatization, there are changes in the estrus cycle of all ten female mice from normal phase to prolonged diestrus. This incident illustrated the Lee-Boot effect of estrus suppression.

\begin{tabular}{ccccccccccccc}
\hline Mouse & Day 1 & Day 2 & Day 3 & Day 4 & Day 5 & Day 6 & Day 7 & Day 8 & Day 9 & Day 10 & Day 11 & Day 12 \\
\hline $\mathbf{1}$ & P & E & D & D & D & D & D & D & D & D & D & D \\
$\mathbf{2}$ & P & E & D & D & D & D & D & D & D & D & D & D \\
$\mathbf{3}$ & E & M & D & D & D & D & D & D & D & D & D & D \\
$\mathbf{4}$ & E & M & D & D & D & D & D & D & D & D & D & D \\
$\mathbf{5}$ & E & M & D & D & D & D & D & D & D & D & D & D \\
$\mathbf{6}$ & E & D & D & D & D & D & D & D & D & D & D & D \\
$\mathbf{7}$ & E & D & D & D & D & D & D & D & D & D & D & D \\
$\mathbf{8}$ & M & D & D & D & D & D & D & D & D & D & D & D \\
$\mathbf{9}$ & D & D & D & D & D & D & D & D & D & D & D & D \\
$\mathbf{1 0}$ & D & D & D & D & D & D & D & D & D & D & D & D \\
\hline
\end{tabular}

Proestrus $=\mathbf{P}$ Estrus $=\mathrm{E}$ Metestrus $=\mathrm{M} \quad$ Diestrus $=\mathrm{D}$

Table 1: Number of mice in each of the four phases of estrus cycle for 12 days of acclimatization

\begin{tabular}{llccc}
\hline & \multicolumn{4}{c}{ Duration of hours of exposure to male urine soaked bedding } \\
\hline Phases & 0 hour & 24 hours & $\mathbf{4 8}$ hours & $\mathbf{7 2}$ hours \\
\hline Proestrus & None & None & 3 mice & 5 mice \\
Estrus & None & None & 1 mouse & 3 mice \\
Metestrus & None & None & None & None \\
Diestrus & All mice & All mice & 7 mice & 2 mice
\end{tabular}

Table 2: Mice in different phases of the estrus cycle upon exposure to male urine soaked bedding for 24 hours, 48 hours and 72 hours. 
Table 2 showed an increase in the number of mice entering the phases of proestrus and estrus associated with the duration of male urine exposure. The findings shown here were modifications in the estrus cycle from prolonged diestrus to proestrus and estrus. In this study, after exposure to male urine soaked-bedding for 48 hours, six female mice were in diestrus, three were in proestrus while one mouse has entered the estrus phase. However, after 72 hours of exposure, two female mice enter proestrus, six female mice has entered the estrus phase while the remaining two female mice which were still in the diestrus phase.

\subsection{Time taken for the Whitten Effect to occur}

From Figure 1, eight out of ten female mice successfully exhibited proestrus and estrus after 72 hours of exposure to the male urine soaked-bedding evident from the vaginal epithelial cell characteristics and vulva appearance from pale and dry to pink and moist. The mice also showed increase excitement when exposed to the male urine soaked-bedding. The highest number of mice exhibited estrus showing the synchronization of estrus in the grouped female mice. These findings suggest that the pheromone in male mice urine can stimulate the estrus in grouped female mice. The time taken for occurrence of the Whitten effect was 72 hours following exposure to male urine soaked-bedding. The statistical test used in this study was the Cochran's Q test. This test was used to observe the changes from diestrus to proestrus and later estrus. The results showed a significant difference $(\mathrm{p}<0.05)$ in the number of mice that successfully enter the proestrus and estrus phases over four time points, $\chi 2(2)=18.857$. The four analysed time point are 0 hours, 24 hours, 48 hours, and 72 hours.

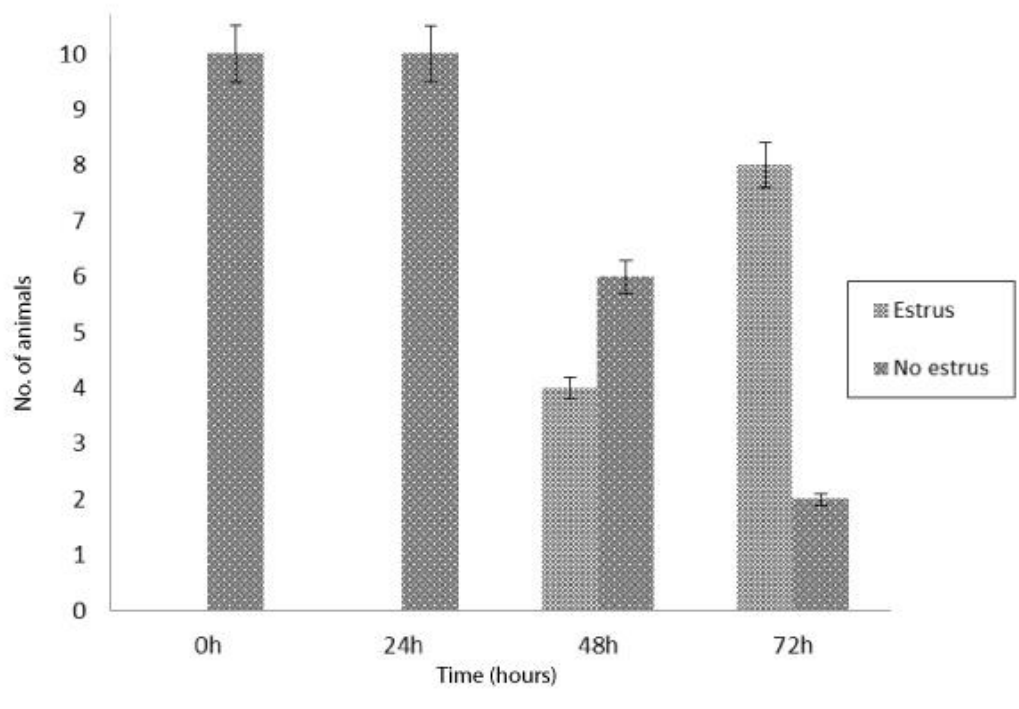

Cochran Q Test: $\chi 2(2)=18.857(\mathrm{p}<0.05)$

Figure 1: Number of mice according to successful rate (estrus) and unsuccessful rate (no estrus) for 24 hours, 48 hours and 72 hours.

\subsection{Discussions}

This study examines the estrus cycle of 10 female mice during 12 days of acclimatization to the animal facility. The normal phase arrangement of the estrous cycle is proestrus, estrus, metestrus, and diestrus. It is necessary to identify the proestrus phase and then pursued by the estrus phase to confirm the estrus cycle is happening. Two methods were used to evaluate the phases of the estrous cycle using visual observation to assess changes of the vulva and vaginal cytology to verify the phases of the estrous cycle. The different phas es of the estrous cycle were observed from vaginal epithelial cell changes and the appearance of the vulva. This study showed that not all of the four phases in ten female mice last for the same period of time. The transitional smears are presently relying on smearing time and it is displaying the features of two successive days of the estrous cycle. The shift from estrus to metestrus offers changes in the appearance of the smear. This is an important point of reference for determining the duration of the cycle and showing the completion of one cycle (estrus) and the start of the next cycle (Caligioni, 2009). Diestrus is the most challenging phase to evaluate due to the relative ratio and variation in cell numbers, is the longest phase and female mice become acyclic and pseudopregnant.

From the findings, after two days of acclimatization to the animal facility, the estrus cycle in ten female mice changes from normal phases to prolong diestrus. Vulva appearance, vaginal cell characteristics, and behaviour of female mice verify the changes in the estrus cycle during grouping together. The female mice showed the Lee-Boot effect in a prolonged diestrus phase after 12 days or 3 cycles of estrus in a cramped cage. It is triggered by the effects of an estrogen-dependent pheromone produced through the urine that acts on the recipients' vomeronasal organ. Urine is the main cause of the chemosignal that suppresses the estrus. The inhibition of the estrous cycle may also occur when the female mouse comes into contact with urine soaked-bedding from other females. This Lee-Boot effect also described the possibility of spontaneous pseudopregnancy in mice.

The Whitten effect is described when pheromones in male mice urine stimulates synchronous estrous when a large number of female mice are housed together in the absence of the male (Petrulis, 2013). Upon exposure to male urine soaked-bedding, changes in the estrus cycle were apparent from prolonged diestrus to proestrus and estrus based on vulva appearance, vaginal cell characteristic and behaviour of the mice. There is a significant difference $(\mathrm{p}<0.05)$ in the number of mice that enter the proestrus and estrus phases successfully over four-time points which are 0 hours, 24 hours, 48 hours and 72 hours using Cochran's Q test. This test was used to observe changes in the number of mice from diestrus to proestrus and later estrus phases. An increase in the number of female mice entering the proestrus and estrus phases was observed after 48 hours of exposure to male urine soaked-bedding. At 72 hours, eight out of ten female mice effectively synchronized estrus as evident from pale and dry vulva to pink and moist vulva appearance with a high number of cornified cells in the vaginal smear. The mice also showed an increased excitement and some specific behavioural response such as lordosis (McCartney and Marshall, 2014). In a similar study on exploratory behaviour in female mice, the role of volatile molecules in adult male mouse urine causes increase lordosis as compared to MUP 
strip of ligands (sMUP). The result from the study indicates that male urine airborne molecules facilitate initial stimulus exploration by female mice, which is efficient at very small doses (Redaelli et al., 2014). The Whitten Effect can be effectively utilized to increase considerably the number of mice required for breeding tests where at the moment of exposure to male urine soaked-bedding the female mice must be in proestrus or estrus (Dalal et al., 2001). The benefit of the Whitten effect using male mice urine soaked-bedding is a straightforward and safe method, affordable and does not demand administration injection.

\subsection{Conclusions}

These easy and reliable methods of vaginal cytology and visual observation can be implemented to determine the estrus cycle phases of the female mice without changing the reproductive status. Furthermore, prolonged diestrus in the mice due to cramp surroundings is helpful in reproductive research for detecting the Lee-Boot effect assessment. The association between pheromone release and female mouse interaction is highlighted by pheromone signals. This research proved that the Whitten effect occurs after 72 hours of exposure to male urine soaked-bedding as observed based on vulva appearance, vaginal cell characteristics, and behavior in grouped female mice. The use of male mice urine soaked-bedding has resulted in considerably enhanced breeding effectiveness in mice.

\subsection{Acknowledgements}

The authors wish to thank the Faculty of Medicine and Health Sciences, UPM Serdang for research grant and facilities at the Physiology Research Laboratory. Nur Hannan Zakaria is a postgraduate student at the same Faculty.

\subsection{Disclosures}

The authors declare no conflicts of interest in this work

\section{0}

\section{References}

Aritonang, T. R., Rahayu, S., Sirait, L. I., Karo, M. B., Simanjuntak, T. P., Natzir, R. Kamelia, E (2017). The Role of FSH, LH, Estradiol and Progesterone Hormone on Estrus Cycle of Female Rats. Journal Of Basic And Applied Research. Vol. 35 (1). 92-100.

Bertolin, K., \& Murphy, B. D. (2014). Reproductive Tract Changes During the Mouse Estrous Cycle. In The Guide to Investigation of Mouse Pregnancy. Academic Press. Pages 85-94.

Bind, R.H., Minney, S.M., Rosenfeld, S. J., and Hallock, R.M. (2013). The Role of Pheromonal Responses in Rodent Behavior: Future Directions for the Development of Laboratory Protocols. Journal of American Association of Laboratory Animal Science. Mar Vol.52 (2): 124-129.

Byers, S. L., Wiles, M. V., Dunn, S. L., \& Taft, R. A. (2012). Mouse Estrous Cycle Identification Tool and Images. PLoS ONE, 7(4). e35538. doi: 10.1371/journal.pone.0035538.

Chamero P, Marton TF, Logan DW, Flanagan K, Cruz JR, Saghatelian A, Cravatt BF, Stowers L. Identification of protein pheromones that promote aggressive behaviour. Nature. 2007. Vol 450:899-902.

Carlson, Neil R. (2013). Physiology of behavior (11th ed.). Boston: Pearson. p. 335. ISBN 0205239390.

Caligioni, C. S. (2009). Assessing Reproductive Status/Stages in Mice. Current Protocols in Neuroscience. doi:10.1002/0471142301.nsa04is48.

Champlin, A. K., Dorr, D. L., \& Gates, A. H. (1973). Determining the Stage of the Estrous Cycle in the Mouse by the Appearance of the Vagina. Biology of Reproduction, 8(4), 491-494.

Dalal, S. J., Estep, J. S., \& Valentin-Bon, I. E., \& Jerse, Ann. E. (2001). Standardization of the Whitten Effect to Induce Susceptibility to Neisseria gonorrhoeae in Female Mice. Contemporary topics in laboratory animal science / American Association for Laboratory Animal Science, 40(2). 13-7.

Ekambaram, G., Sampath Kumar, S. K., \& Joseph, L. D. (2017). Comparative Study on the Estimation of Estrous Cycle in Mice by Visual and Vaginal Lavage.Method. Journal Of Clinical And Diagnostic Research. doi:10.7860/jcdr/2017/23977.9148.

Gonzalez, G. (2016). Determining the Stage of the Estrous Cycle in Female Mice by Vaginal Smear. Cold Spring Harbor Protocols, 2016(8). doi: $10.1101 /$ pdb.prot094474.

Grammer, K., Fink, B., and Neave, N. (2005). Review on Human Pheromones and Sexual Attraction. European Journal of Obstetrics and Gynaecology and Reproductive Biology. Vol. 118 (2), pp:135-142.

Haga S, Hattori T, Sato T, Sato K, Matsuda S, Kobayakawa R, Sakano H, Yoshihara Y, Kikusui T, Touhara K. (2010). The male mouse pheromone ESP1 enhances female sexual receptive behaviour through a specific vomeronasal receptor. Nature. Vol. 466:118-22.

Hellier, V., Brock, O., Candlish, M., Desroziers, E., Aoki, M., Mayer, C., Piet, R. Herbison, A., Colledge, WH., Prevot, V Boehm U., and Bakker, J. (2018). Female Sexual Behaviour In Mice is Controlled by Kisspeptin Neurons. Nature Communications. Vol. 9 (400).

Macdonald, J. K., Pyle, W. G., Reitz, C. J., \& Howlett, S. E. (2014). Cardiac contraction, calcium transients, and myofilament calcium sensitivity fluctuate with the estrous cycle in young adult female mice. American Journal of Physiology-Heart and Circulatory Physiology, 306(7). doi:10.1152/ajpheart.00730.2013.

Maffucci, J. A., \& Gore, A. C. (2009). Chapter 2 Hypothalamic Neural Systems Controlling the Female Reproductive Life Cycle. International Review of Cell and Molecular Biology, 69-127. doi:10.1016/s1937-6448(08)02002.

Mamrot, J., Pangestu, M., Walker, D., Gardner, D. K., \& Dickinson, H. (2015). Confirmed dioestrus in pseudopregnant mice using vaginal exfoliative cytology improves embryo transfer implantation rate. Reproductive BioMedicine Online, 31(4).

Mclean, A. C., Valenzuela, N., Fai, S., \& Bennett, S. A. (2012). Performing Vaginal Lavage, Crystal Violet Staining, and Vaginal Cytological Evaluation for Mouse Estrous Cycle Staging Identification. Journal of Visualized Experiment.

McCartney, C. R., Marshall, J. C. (2014). Chapter 1 - Neuroendocrinology of Reproduction .

Yen \& Jaffe's Reproductive Endocrinology ( $7^{\text {th }}$ Edition), https://doi.org/10.1016/B978-1-4557-2758-2.00001-9.

Petrulis, A. (2013). Chemosignals, hormones and mammalian reproduction. Hormones and Behavior, Vol. 63(5), 723-741. doi:10.1016/j.yhbeh.2013.03.011

Redaelli, M., Orsetti, A., Zagotto, G., Cavaggioni, A., \& Mucignat-Caretta, C. (2014). Airborne molecules released from male mouse urine affect female exploratory behavior. Frontiers in Ecology and Evolution, 2. doi:10.3389/fevo.2014.00028. 
Roberts, S.A., Simpson, D.M., Armstrong, S.D,, Davidson, A.J., Robertson, D.H., McLean, L., Beynon, R.J., Hurst, J.L. (2010). Darcin: a male pheromone that stimulates female memory and sexual attraction to an individual male's odour. BMC Biol. Vol. 8:75.

Roberts SA, Davidson AJ, McLean L, Beynon RJ, Hurst JL. (2012). Pheromonal induction of spatial learning in mice. Science. Vol.338:14625.

Rodriguez, I. (2016). Chapter 10. Vomeronasal Receptors: V1Rs, V2Rs and FPRs. The Detection of Odors, Tastes and other Chemostimuli. In Chemosensory Transduction. pp:175-190. https://doi.org/10.1016/B978-0-12-801694-7.00010-X.

Thoß, M., Luzynski, K. C., Enk, V. M., Razzazi-Fazeli, E., Kwak, J., Ortner, I., \& Penn, D. J. (2019). Author Correction: Regulation of volatile and non-volatile pheromone attractants depends upon male social status. Scientific Reports, 9(1). doi: 10.1038/s41598-019-41666$4: 10.1016 / j . c o p h y s .2018 .10 .001$.

Vandenbergh, J.G. (2008) The house mouse in biomedical research. In: Book of Models for Biomedical Research. PM Conn, ed., Humana Press, Totowa, NG. Pages 187-190.

Wani, A.A., Dhindsa, S.S., Shafi, T.A., Chowdhary, S.R.A. and Balwinder, K. (2013). The role of pheromones in animal reproduction - a review. Progressive Res., 8: 14-18.

Yoshinaka, K., Yamaguchi, A., Matsumura, R., Node, K., Tokuda, I., \& Akashi, M. (2017). Effect of different light-dark schedules on estrous cycle in mice, and implications for mitigating the adverse impact of night work. Genes to Cells, Vol.22(10), 876-884. doi:10.1111/gtc. 12522 